\title{
REVIEW WORK ON ANALYSIS OF F1 CAR FRAME USING ANSYS
}

\author{
N. G. Jogi', Akshay P. Take², Yogesh Asolkar ${ }^{3}$, Sheikh M Aftab ${ }^{4}$ \\ ${ }^{1}$ Guide, Mechanical Engineering, J.D.I.E.T. Yavatmal, Maharashtra, India \\ ${ }^{2}$ Student, Mechanical Engineering, J.D.I.E.T. Yavatmal, Maharashtra, India \\ ${ }^{3}$ Student, Mechanical Engineering, J.D.I.E.T. Yavatmal, Maharashtra, India \\ ${ }^{4}$ Student, Mechanical Engineering, J.D.I.E.T. Yavatmal, Maharashtra, India
}

\begin{abstract}
SAE Supra India organizes the undergraduate racing competition for 2013 in which teams are challenged to design considering the various circumstances and to fabricate the making a design in the campus itself. This paper's sole focus is the design and analysis for a chassis which has to sustain the racing environment. As chassis plays a vital role in a race car and also it is the back bone of a good race car. Good designs allow a light, stiff and extremely safe chassis to be produced at. The work shown in this research paper was taken participation by Black Hawk SAE Supra team.

This paper introduces several concepts of frame's load distributions such a lateral, longitudinal vertical and horizontal torsion and consequent deformation modes. .Various studies were conducted upon the understanding the relation of the machine elements with the user according to Ergonomics consideration. Ergonomics considers factors such as Drivers visibility, seat inclination, thermal isolation etc.

The chassis design was carried out on CAD software such as Pro-Engineers. Design model was prepared using anthropometric parameters of tallest driver as 95th percentile male was selected to SAE rules book. Static and dynamic load distributions were calculated analytically followed by extensive study of various boundary conditions to be applied during diverse FEA (Finite Element Analysis) test which was carried out in Ansys. Stress distributions, lateral displacements during static, dynamic and frequency modes were analyzed and found considerably high factor of safety as 3.85 .
\end{abstract}

Keywords: FAE (Finite Element Analysis)

\section{INTRODUCTION}

SAE (society of automotive engineers ) has conduct the competition in which student build, design, and compete with a small formula race car these compensation help in providing the educational experience that analogous to the type of project work they will face in work force ,it also help the student to participate in group work ,project management and finance have been incorporated in the rules of SAE. The SAE competition is consisting of static and dynamic event where they are judge on the performance of vehicle.

The main philosophy behind the impact test is that to assure the driver safety in the survival cell the survival cell should be strong enough to absorb the force and to distribute the force from front to the back without large deflection to the chassis. The chassis should be strong enough to absorb the energy when front, back, side, torsional are the load which occur during the impact test should be distributed progressively. These paper give the introduction of the various frame design concept and provide analytical and the experimental both. The various loading condition are also describe below.
This paper is consisting of various tests which are conduct on the chassis which give the detail description are documented below. Which are such as front impact test, side impact test, roll over test, torsional test these are the test which are conducted. The paper has also provide the detail description of the material which is used while design of the vehicle these has widen the structure strength and modeled the design to inculcate more driver comfort ,safety the structure is in the truss form which help to reduce the energy in structure.

The mode of load distribution and the concept were taken from the various books and the reference.

The following paragraph show the relevant result and the studies conducted on the performance and analysis done by them are describe in below paragraph of the literature.

Ravinder Pal Singh[1] carried out study to determine the effect on the frame which was used for the analysis. The catia software was used for the modelling and the ansys software was used for the analysis of the chassis. The chassis was passed through the various test which was conducted by them for e.g. static Stress distributions, lateral displacements during 
static, dynamic loading etc the test were done by them to determine the factor of safety of the frme should be in th safe mode. They had compared the new chassis with the older chassis and the weight of the chassis was reduced from $36 \mathrm{~kg}$ to $32 \mathrm{~kg}$ of the older chassis and the torsional rigidity of the chassis was improved from 2.64 times the older chassis.

Chassis was found to be safe significantly in static (bending) and dynamic (acceleration) modes with stress values noticeably less than the yield strength. Critical value of stress was found to be $5.53 \times 1010 \mathrm{~N} / \mathrm{m} 2$ at a frequency of $156.86 \mathrm{~Hz}$. Although below this frequency, chassis was found to

be safe and exhibited almost no stress. Dynamometer testing of previous car at approximate $9000 \mathrm{rpm}$ with speed of 90 $\mathrm{km} / \mathrm{h}$, the maximum vibration frequency was noted to be not more than $100 \mathrm{~Hz}$. So, it was expected that vibration range under same conditions will either remain same or decrease for the present design model. Hence chassis was expected to perform well in motion also.

Bryan Chu Oliver Jetson[2] This project will involve examining the front crash structure used to protect the driver in the event of a frontal impact. At the moment every manufacturer has a different method of producing their crash structure, and none of these designs have been officially tested. Freedom is given to the student design groups to develop a new crash box which will be tested against a set of impact requirements which have been based off similar tests used for Formula 1 and Formula 3. The end result of these new designs may be used as new structure which will be common amongst all manufacturers. Ultimately the final product of the project should be a crash structure which can survive these impact tests and be suitable for the low-budget mindset of the Formula Ford series.

We have demonstrated the crash worthiness of a very innovative material. The use of Degussa's Rohacell high density polymer foams is definitely recommended for motorsport crash applications and especially in the low cost environment of Formula Ford. The Rohacell foam exhibits the following upsides homogenous structure, isotropic properties and therefore good off-axis loading tolerance, high Specific Energy Absorption, very easy manufacturing well adapted for small manufacturers. very easy manufacturing well adapted for small manufacturers.

Abrams, Ryan[3] simulation and testing, the objective of this report was to determine the methods and validate results for using an engine as a structural member of a Formula SAE race car chassis. Modal analysis was used with a beam mesh of the chassis to validate an increase of chassis stiffness. Results confirmed that providing structural support at engine mounting points of the chassis increased the stiffness and changed the first elastic mode of vibration from bending to torsion. Additionally a static analysis was used to measure chassis deflection, and established a decrease in deflection when structural support was provided at the engine mounting points of the chassis. This report's conclusion indicates that using the engine as structural member significantly improves the stiffness of the chassis. Furthermore, with improved software Dynamic testing on the frame and engine block should be analyzed to obtain measurable results, establish the allowable forces on the engine and the direction of their application.

Chartree Sithananun,Nuksit Noomwongs[4] they analysed that requirements for the frame are mass less than $30 \mathrm{~kg}$ and torsional stiffness of the whole car more than $1200 \mathrm{Nm} / \mathrm{deg}$, which was an important values that determine the frame performance level. The design is generated using the computer aided program, CATIA, and then the finite element analysis (FEM) is performed in order to determine the mass and torsional stiffness of the frame. After analysis, the frame is constructed and the torsional test is performed to determine the torsional stiffness of the frame. The results are compared and the 2011 frame is decided. The 2011 frame has torsional stiffness of $1030 \mathrm{Nm} / \mathrm{deg}$ and with the mass of $29.8 \mathrm{~kg}$ which accomplish the requirements of the frame. monocoque skins allows strength, stiffness and flexibility to be controlled in different directions. A space frame or space structure is a truss-like, lightweight rigid structure constructed from interlocking struts in geometric pattern. Space frames are series of tubes which are joined together to form a structure.

William B. Riley and Albert R. George[5] The paper introduces several of the key concepts of frame design both analytical and experimental. The different loading conditions and requirements of the vehicle frame are first discussed focusing on road inputs and load paths within the structure This model examines the frame and overall chassis torsional stiffness relative to the suspension spring and anti-roll bar rates. A finite element model is next developed to enable the analysis of different frame concepts. Some modeling guidelines are presented for both frames in isolation as well as the assembled vehicle including suspension. They tested on different road loads and deformation modes were considered as well as some generic design targets based on experience and strain gauged suspension links. A simple mathematical model was developed for comparing the structural stiffness to the suspension stiffness to gain insight into proper design targets for the vehicle structure.

Mohd Azman. Abdullah, Muhd Ridzuan. Mansor, [6] they have used catia v5 version for the modelling of the chassis and the finite element of analysis they have used ASTM 500 material hot steel square cross section rod were used for the fabrication of the frame simulation analysis results have established approving outcome in term of factor of safety at different loading tests. The final products for both designs have been fabricated successfully and verified the practice of design for manufacture (DFM). The fitting and assembly of 
the rolling chassis have verified the accuracy and acceptable tolerance in design.

\section{CONCLUSIONS}

There is much software on which we can work and design the model such as solid works, catia, pro-e, etc can use for modelling and for it can be done by using finite element analysis tool for the frame.

It can be conclude that the less stress distribution can be seen when the frame has a triangular bar arrangement thought the chassis. The deflection in the frame is reduced by these arrangements and the load of the chassis can be reduced.

From the above study we conclude that different material can be used for the design of frame, material which can be used are, AISI 4013,monocoque etc. These materials are used for reduction of the weight and increasing the strength of the chassis. The factor of safety should be greater than 2 so as the frame should withstand the racing condition.

\section{REFERENCES}

[1] Ravinder Pal Singh," Structural performance analysis of formula sae car, Jurnal Mekanikal December 2010, No. 31, 46 - 61

[2] Bryan Chu Oliver Jetson," Crash Absorption Structure for Formula Ford Use of Rohacell in Motorsport Crash Worthiness

[3] Abrams, Ryan," Formula sae race car analysis: simulation \& testing of the engine as a structural member", F2008 SC-005

[4] Chartree Sithananun,Nuksit Noomwongs," SAE Student Formula Space Frame Design and Fabrication", The Second TSME International Conference on Mechanical Engineering 19-21 October, 2011, Krabi

[5] William B. Riley and Albert R. George,” Design, Analysis and Testing of a Formula SAE Car Chassis", SAE TECHNICAL PAPER SERIES 2002-01-3300

[6] Mohd Azman. Abdullah, Muhd Ridzuan. Mansor," Design, Analysis and Fabrication of Chassis Frame for UTeM Formula VarsityTM Race Car", International Journal of Mining, Metallurgy \& Mechanical Engineering (IJMMME) Volume 1, Issue 1 (2013) ISSN 2320-4060 (Online) 